\title{
Neisseria meningitidis ST11 Complex Isolates Associated with Nongonococcal Urethritis, Indiana, USA, 2015-2016
}

\author{
Evelyn Toh, ${ }^{1}$ Dharanesh Gangaiah, ${ }^{1}$ \\ Byron E. Batteiger, James A. Williams, \\ Janet N. Arno, Albert Tai, Teresa A. Batteiger, \\ David E. Nelson
}

At a clinic in Indianapolis, Indiana, USA, we observed an increase in Neisseria gonorrhoeae-negative men with suspected gonococcal urethritis who had urethral cultures positive for $N$. meningitidis. We describe genomes of 2 of these $N$. meningitidfiis sequence type 11 complex urethritis isolates. Clinical evidence suggests these isolates may represent an emerging urethrotropic clade.

$N$ Teisseria meningitidis and $N$. gonorrhoeae are exclusive human pathogens. N. meningitidis is a leading cause of sepsis and meningitis, whereas $N$. gonorrhoeae (gonococcus) traditionally causes gonorrhea, a sexually transmitted infection involving the genitals, rectum, and throat. These species usually occupy distinct niches but may cause reciprocal diseases when $N$. meningitidis colonizes the anogenital tract or gonococcus colonizes oropharyngeal mucous membranes $(1,2)$.

Sporadic cases of meningococcal urethritis have been reported since the 1930 s (3). It was recognized by the 1970s that urethral $N$. meningitidis infections could be spread by oral sex and were more common in men who have sex with men (4). Most $N$. meningitidis urethritis cases identified before 1993 were caused by strains from serogroups A and B (5), but outbreaks involving other serogroups and nontypeable strains have been reported (610). These cases have typically presented with purulent urethritis or proctitis with gram-negative intracellular diplococci (GNID) identified by Gram stain of urethral exudates. The current prevalence of meningococcal urethritis is unknown in the United States but was thought to be low (4). However, a recent spike in heterosexual N. meningitidis urethritis cases beginning in 2013 in Ohio and Michigan, reported by Bazan et al. in association with the Gonococcal Isolate Surveillance Project, was linked to a nongroupable clonal $N$. meningitidis strain (sequence type 11 [ST11], clonal complex [CC] ET-37) (11). Whether N. meningitidis ST11 strains are common causes of non-

Author affiliations: Indiana University School of Medicine, Indianapolis, Indiana, USA (E. Toh, D. Gangaiah, B.E. Batteiger, J.A. Williams, J.N. Arno, T.A. Batteiger, D.E. Nelson); Tufts University School of Medicine, Boston, Massachusetts, USA (A. Tai).

DOI: http://dx.doi.org/10.3201/eid2302.161434 gonococcal urethritis or if these strains have adaptations that enhance their virulence for the urethra is unknown because genomes of urethral $N$. meningitidis isolates have not been previously reported.

Beginning in early 2015, we observed that $4(6 \%)$ of 59 men we enrolled in gonococcus treatment trials in our clinic in Indianapolis, Indiana, USA, and who had urethral specimens that were positive on Gram stain tested negative for gonococcus by specific nucleic acid amplification tests (NAATs). Sugar fermentation reaction profiles of the isolates from all 4 of these men suggested that they were infected with $N$. meningitidis. We describe the recent epidemiology of these suspected $N$. meningitidis urethritis cases and the genomes of 2 of these $N$. meningitidis isolates.

\section{The Study}

We enrolled 59 men (age range 20-61 years, median age 31 years) in 3 gonoccocal treatment trials from April 2014 through February 2016 at the Bell Flower Clinic, a public sexually transmitted infections clinic, in Indianapolis, Marion County, Indiana. All of these men had purulent urethral discharge with $>10$ leukocytes and GNID on Gram stains of urethral exudate. However, 4 men tested negative for gonococcus with specific NAATs (APTIMA Combo 2, GenProbe Inc., San Diego, CA, USA; or COBAS 4800, Roche Diagnostics, Indianapolis, IN, USA). All 4 men reported recent vaginal and oral sexual exposures. Urethral cultures yielded growth with colony morphology, oxidase, and Gram stain results consistent with gonococcus, but the isolates fermented glucose and maltose but not lactose, consistent with $N$. meningitidis. A pharyngeal swab from 1 of these men also grew $N$. meningitidis, whereas rectal swab specimens from all 4 were culture negative. All 4 cases responded to investigational gonococcal antibiotic regimens and were culture plus NAAT negative by test of cure assessed at day 7 posttreatment. Two of these isolates (NM1 and NM2), which were susceptible to ampicillin, ceftriaxone, chloramphenicol, levofloxacin and meropenem, were subcultured and confirmed to be $N$. meningitidis by matrix-assisted laser desorption/ionization time-of-flight mass spectrometry.

Whole-genome sequencing of NM1 and NM2 (online Technical Appendix, http://wwwnc.cdc.gov/EID/ article/23/2/16-1434-Techapp1.pdf) revealed that these 2 isolates closely resembled strains of ST11 and CC11, with fine type PorA VR 1.5-1, PorA2 10-8; FetA3-6 ST11 (CC11).

${ }^{1}$ These authors contributed equally to this article. 


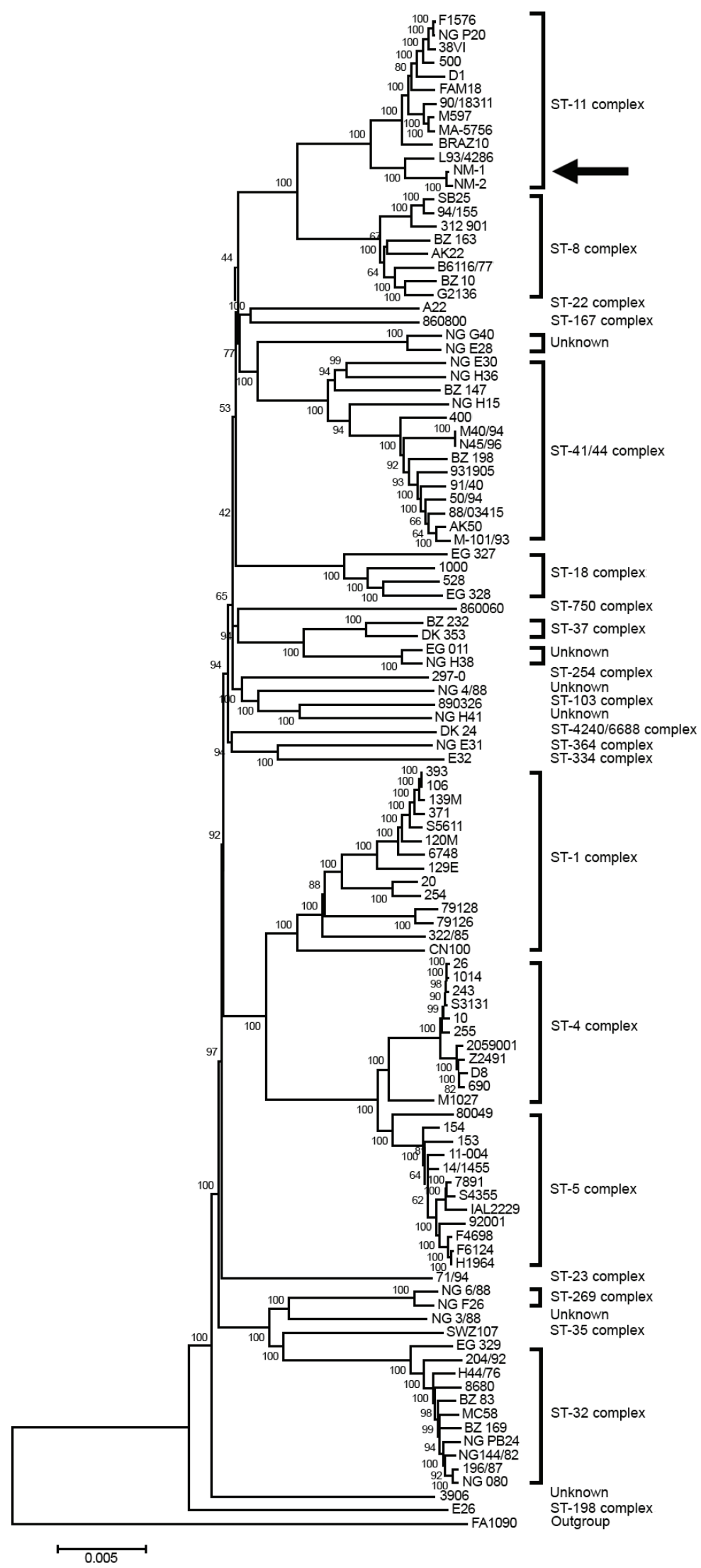

Figure. Rooted phylogenetic tree of Neisseria meningitidis sequence type 11 urethral isolates from men in Indianapolis, Indiana, USA, 2015-2016, compared with representative serogroup strains of $\mathrm{N}$. meningitidis. Tree was inferred by using the neighbor-joining method constructed with MEGA7 (13). The percentage of replicate trees in which the associated taxa clustered together in 500 bootstrap tests is indicated next to the branches. The tree is drawn to scale, and branch lengths correspond to evolutionary distances used to infer the phylogenetic tree. Arrow indicates urethritis strains NM1 and NM2. GC strain FA1090 was used as the outgroup. Scale bar indicates nucleotide substitutions per site. 
Phylogenetic analysis indicated that NM1 and NM2 likely share a common ancestor with NM L93/4286, a serogroup C invasive strain from the United Kingdom (12). A rooted tree constructed by the neighbor-joining method indicated that NM1 and NM2 are closely related to $N$. meningitidis serogroup C strain, FAM18 (Figure) (13). Gene-to-gene comparison with FAM18 loci revealed that 29 out of 1,975 loci were missing in $\geq 1$ of the urethral isolates, although 24 of these were near the end of a contig in either NM1 or NM2. Of the remaining loci, 1,075 (56\%) were identical in all three strains, and 1,848 (96\%) were identical in NM1 and NM2.

The capsule locus encompasses $\approx 24 \mathrm{~kb}$ in most NM isolates and contains genes that mediate capsule synthesis, transport, assembly and translocation, as well as LPS synthesis. NM1 and NM2 lack $\operatorname{css} C, \operatorname{css} B$, and $\operatorname{css} A$ (former designation siaC, siaB, and siaC [14]), whereas these are in- tact in NM FAM18 and MC58 (online Technical Appendix). Our analysis indicated that these genes are highly conserved in diverse isolates from $N$. meningitidis serogroups $\mathrm{B}, \mathrm{C}, \mathrm{W}$, and $\mathrm{Y}$, but are also absent in gonococcal strain FA1090.

To estimate the prevalence of presumptive NM urethritis at $\mathrm{BFC}$, we retrospectively examined 107 cases from men seen between 2013 and 2016 who had Gram stain results positive for WBCs and GNID but who had negative GC NAATs. Rates of negative GC NAATs among men with positive Gram stains were 12/436 $(2.8 \%)$ in $2013,8 / 552(1.4 \%)$ in $2014,37 / 533(6.9 \%)$ in 2015 , and $50 / 510(9.8 \%)$ in the first 3 quarters of 2016 , indicating a significant increase in these cases during the study interval by both a $\chi^{2}$ test $(p<0.0001)$ comparing the 4 years and the Mantel-Haenszel test for a trend over time $(p=0.005)$. Analysis of cases before 2013 was not

\begin{tabular}{|c|c|c|}
\hline \multirow[b]{2}{*}{ Characteristic } & \multicolumn{2}{|c|}{ No. (\%) persons } \\
\hline & 2015 & 2016 \\
\hline \multicolumn{3}{|l|}{ Race } \\
\hline White & $6(16)$ & $5(10)$ \\
\hline Black & $29(78)$ & $45(90)$ \\
\hline Other & $2(5)$ & 0 \\
\hline \multicolumn{3}{|l|}{ Ethnicity } \\
\hline Non-Hispanic & $35(95)$ & $50(100)$ \\
\hline Hispanic & $2(5)$ & 0 \\
\hline \multicolumn{3}{|l|}{ Self-identified sexual orientation } \\
\hline Heterosexual & $34(92)$ & $48(96)$ \\
\hline Homosexual & $2(5)$ & $1(2)$ \\
\hline Bisexual & $1(3)$ & 0 \\
\hline \multicolumn{3}{|l|}{ Modes of sexual contact $\dagger$} \\
\hline Insertive vaginal intercourse & $33(89)$ & $45(90)$ \\
\hline Insertive oral sex & $35(95)$ & $44(88)$ \\
\hline Receptive oral sex & $19(51)$ & $33(66)$ \\
\hline Insertive anal intercourse & $1(3)$ & $1(2)$ \\
\hline Receptive anal intercourse & $1(3)$ & $1(2)$ \\
\hline \multicolumn{3}{|l|}{ Symptoms } \\
\hline Discharge & $35(95)$ & $49(98)$ \\
\hline Dysuria & $2(5)$ & 0 \\
\hline Discharge/dysuria & $37(100)$ & $49(98)$ \\
\hline No discharge/dysuria & 0 & $1(2) \ddagger$ \\
\hline \multicolumn{3}{|l|}{ Most recent HIV status } \\
\hline Negative, documented or self-reported & $32(86)$ & $45(90)$ \\
\hline Not tested and unknown & $5(14)$ & $5(10)$ \\
\hline \multicolumn{3}{|l|}{ Exchange sex for drugs or money } \\
\hline Yes & $2(5)$ & 0 \\
\hline No & $33(89)$ & $49(98)$ \\
\hline Unknown & $2(6)$ & $1(2)$ \\
\hline \multicolumn{3}{|c|}{ Noninjection recreational drug use, excluding alcohol, preceding $60 \mathrm{~d}$} \\
\hline Yes & $17(46)$ & $31(62)$ \\
\hline No & $18(49)$ & $19(38)$ \\
\hline Unknown & $2(5)$ & 0 \\
\hline \multicolumn{3}{|l|}{ Treatment provided } \\
\hline Ceftriaxone plus azithromycin & $34(92)$ & \\
\hline Ceftriaxone plus doxycycline & $1(3)$ & 0 \\
\hline Other & $2(5)$ & $1(2)$ \\
\hline \multicolumn{3}{|c|}{ Urethral coinfection with Chlamydia trachomatis by NAAT } \\
\hline Positive & $6(16)$ & $8(16)$ \\
\hline Negative & $31(84)$ & $42(84)$ \\
\hline
\end{tabular}

*NAATs, nucleic acid amplification tests.

†One man had none.

†Had irritated meatus. 
possible because of the lack of discrimination in the NAAT used at the clinic.

Similar to the case-patients recently reported from Ohio and Michigan (11), our 37 case-patients in 2015 had an average age of 32 (range 20-61) years and were symptomatic with either discharge $(\mathrm{n}=35)$ or dysuria $(\mathrm{n}=2)$ and predominantly heterosexual $(\mathrm{n}=34)$ (Table). Most received oral sex $(n=35)$ and were HIV negative $(n=32 / 32$ tested).

\section{Conclusions}

Together, our results and those of Bazan (15) suggest that $N$. meningitidis ST11 could be a notable emerging cause of nongonococcal urethritis; more extensive sequencing and comparisons of recent $N$. meningitidis ST11 isolates from around the United States are underway. However, our results support the observation that both the rates and geographic distribution of $N$. meningitidis-associated urethritis cases in the United States are increasing. Sequencing additional isolates should clarify whether these isolates correspond to an emerging urethrotropic clade of $N$. meningitidis. Retrospective comparisons of NAAT-negative, GNID-positive urethritis case rates might also help discern whether these cases have been increasing elsewhere. As fewer clinics perform routine Gram staining, a serious concern is that infections with urethral discharge, coupled with a negative gonococcusspecific NAAT, could be misdiagnosed as a Chlamydia or Trichomonas infection. We speculate that an increased number of $N$. meningitidis cases may occur if we limit our clinical diagnoses solely on the results of current diagnostic methods, thereby causing asymptomatic cases to go untreated. Therefore, continued investigation into diagnostic methods targeting urethral specific $N$. meningitidis isolates is pressing, to control the transmission of sexually transmitted $N$. meningitidis. Finally, we note that because the meningococcal ctr $A$ gene is highly conserved in NM1 and NM2, they should be able to be differentiated from gonococcus by using meningococcal ctrA reverse transcription PCR assays (15).

\section{Acknowledgments}

We thank Rosemary Batteiger for assistance with mass spectrometry. We also thank the Bell Flower Clinic staff for the enrollment of patients into the gonorrhea study and for their meticulous documentation of clinical characteristics of patients. In addition, we are grateful to Stan Spinola for his advice and review of this manuscript.

This work was supported in part by National Institutes of Health grant no. AI116706-01A1 (to D.E.N. and B.E.B.).

Dr. Toh is a postdoctoral fellow at the Indiana University School of Medicine in Indianapolis, Indiana. Her research interests include pathogen discovery using cultivation-independent metagenomic sequencing and comparative genomic approaches and molecular typing of sexually transmitted bacterial pathogens.

\section{References}

1. Feldman HA. Meningococcus and gonococcus: never the twain - well, hardly ever. N Engl J Med. 1971;285:518-20. http://dx.doi.org/10.1056/NEJM197108262850914

2. Carpenter CM, Charles R. Isolation of meningococcus from the genitourinary tract of seven patients. Am J Public Health Nations Health. 1942;32:640-3. http://dx.doi.org/10.2105/AJPH.32.6.640

3. Murray E. Meningococcus infections of the male urogenital tract and the liability to confusion with gonococcus infection. Urol Cutaneous Rev. 1939;43:739-41.

4. Janda WM, Bohnoff M, Morello JA, Lerner SA. Prevalence and site-pathogen studies of Neisseria meningitidis and $N$. gonorrhoeae in homosexual men. JAMA. 1980;244:2060-4. http://dx.doi.org/ 10.1001/jama.1980.03310180026026

5. Nebreda T, Campos A, Merino FJ. Urethritis caused by Neisseria meningitidis serogroup C. Clin Microbiol Infect. 1999;5:57-59. http://dx.doi.org/10.1111/j.1469-0691.1999.tb00101.x

6. Rodriguez CN, Rodriguez-Morales AJ, Garcia A, Pastran B, Rios A, Calvo A, et al. Quinolone and azithromycin-resistant Neisseria meningitidis serogroup C causing urethritis in a heterosexual man. Int J STD AIDS. 2005;16:649-50. http://dx.doi.org/10.1258/0956462054944363

7. Gregory JE, Crook R, Keeler G. Urethritis attributable to Neisseria meningitidis, group X: a case report. J Natl Med Assoc. 1979;71:845-6.

8. Hayakawa K, Itoda I, Shimuta K, Takahashi H, Ohnishi M. Urethritis caused by novel Neisseria meningitidis serogroup $\mathrm{W}$ in man who has sex with men, Japan. Emerg Infect Dis. 2014;20:1585-7. http://dx.doi.org/10.3201/eid2009.140349

9. Hagman M, Forslin L, Moi H, Danielsson D. Neisseria meningitidis in specimens from urogenital sites. Is increased awareness necessary? Sex Transm Dis. 1991;18:228-32. http://dx.doi.org/10.1097/00007435-199110000-00006

10. Shanmugaratnam K, Pattman RS. Acute urethritis due to Neisseria meningitidis. Genitourin Med. 1989;65:401-2.

11. Bazan JA, Peterson AS, Kirkcaldy RD, Briere EC, Maierhofer C, Turner AN, et al. Notes from the field: Increase in Neisseria meningitidis-associated urethritis among men at two sentinel clinicsColumbus, Ohio, and Oakland County, Michigan, 2015. MMWR Morb Mortal Wkly Rep. 2016;65:550-2.

http://dx.doi.org/10.15585/mmwr.mm6521a5

12. Bratcher HB, Corton C, Jolley KA, Parkhill J, Maiden MC. A gene-by-gene population genomics platform: de novo assembly, annotation and genealogical analysis of 108 representative Neisseria meningitidis genomes. BMC Genomics. 2014;15:1138. http://dx.doi.org/10.1186/1471-2164-15-1138

13. Kumar S, Stecher G, Tamura K. MEGA7: Molecular Evolutionary Genetics Analysis version 7.0 for bigger datasets. Mol Biol Evol. 2016;33:1870-4. http://dx.doi.org/10.1093/molbev/ msw054

14. Harrison OB, Claus H, Jiang Y, Bennett JS, Bratcher HB, Jolley KA, et al. Description and nomenclature of Neisseria meningitidis capsule locus. Emerg Infect Dis. 2013;19:566-73. http://dx.doi.org/10.3201/eid1904.111799

15. Corless CE, Guiver M, Borrow R, Edwards-Jones V, Fox AJ, Kaczmarski EB. Simultaneous detection of Neisseria meningitidis, Haemophilus influenzae, and Streptococcus pneumoniae in suspected cases of meningitis and septicemia using real-time PCR. J Clin Microbiol. 2001;39:1553-8. http://dx.doi.org/10.1128/JCM.39.4.1553-1558.2001

Address for corrrespondence: David E. Nelson, Indiana University School of Medicine, Department of Microbiology \& Immunology, 635 Barnhill Dr, MS420, Indianapolis, IN 46202, USA; email: nelsonde@indiana.edu 